\title{
Iterative Computational Scheme of Studying Electromagnetic Wave Propagation through Dielectric Thin Film Medium
}

\author{
E.I. Ugwu ${ }^{1}$ and C.E. Okeke ${ }^{2}$ \\ ${ }^{1}$ Department of Industrial Physics, Ebonyi State University Abakaliki \\ ${ }^{2}$ Departments of Physics and Astronomy University of Nigeria, Nsukka \\ ugwuei@yahoo.com
}

\begin{abstract}
We present an approach to the computation of electromagnetic wave propagation through a dielectric thin film medium using iterative scheme. We used the Green's function technique involving some necessary boundary condition to solve the scalar wave equation. Non-vectorial aspects of the propagating wave through the thin film resulting from the film orientation were considered. The iterative numerical scheme based on the parallel use of Lippmann-Schwinger and Dyson's equations is demonstrated and used judiciously in the computation. The influence of the numerical parameters such as Green's function, thickness of the thin film, dielectric perturbation, $\Delta \varepsilon_{p}(z)$ and mesh size, $N_{p}$ on the propagating wave for three region of electromagnetic wave were clearly assessed.
\end{abstract}

Keywords: Iterative Scheme, Lippmann-Schwinger Equation, Dyson's equation, electromagnetic wave, Thin film, dielectric medium, Green's function, computation, wave equation, and discretization

\section{INTRODUCTION}

For long, significant work and progress had been made in the area of beam propagation method of electromagnetic wave propagation through materials [Feit and Fleck 19978, 1979, 1980]. And since then the assessment of its applicability [van-Roey et al, 1981, Thyle 1 1982], the method has really became the most widely used technique of modeling integrated optics. Though the method based on this concept had its deficiency due to the vectoral nature involved in electromagnetic wave propagation, based on the fact that development of the beam propagation method had two directions. One being with the view of overcoming the limitation of the paraxial approximation [Chu and chaudhuri, 1989; Yevick and Glasner, 1990] in which the attention was to device an exact solution of the scalar wave. The second approach was in a case where the semivectorial method was applied in which the treatment of various components of the electric field were considered simultaneously, but not the coupling among these components[Splett et al,1991, Stern,1991] After these development, vectorial method that involved modeling of vectorial effects such as polarization within the limited frame work the paraxial approximation was evolved [Claudberg et al, 1991, Huang et al, 1991,Martin et al, 1991, Chung et al1991, Liu and Gomelsky, 1992, Huang et al 1992]. Another approach was also proposed to demonstrate a simpler method could be to solve exactly the wave equation for arbitrary dielectric media.[Martin et al 1994]. Recently computation of field propagation through an inhomogeneous thin film medium been demonstrated in which the influence of dielectric 
function and small change in refractive index of the thin film medium was studied [Ugwu, 2011and Ugwu et al 2007] with the problem the direct numerical integration due to the implicit nature of the key equations were pointed out. In this paper therefore, we propose an approach to overcome this problem which is based on iterative scheme on Green's function, Lippmann-Schwinger and Dyson's equations to be involved in order to solve for the field propagation through a dielectric thin film medium. In this case, we first of all obtain the Green's function associated with the scalar wave equation and use it to spell out the value of the field propagating through the thin film that involves Lippmann-Schwinger equation and its counterpart, Dyson's equation which in each case is being iterated for use in obtaining the field profile through the film.

\subsection{THEORETICAL PROCEDURE}

The aim here is to find the solution $\psi(z)$ of the scalar wave equation given as

$$
\nabla^{2} \psi(z)+\omega^{2} \mu_{o} \varepsilon_{o} \varepsilon_{p}(z) \psi(z)=0
$$

For an arbitrary complex dielectric medium $\varepsilon_{p}(z)$ of the homogeneous thin film medium, we assume that it is split into two parts: a homogeneous reference part of dielectric constant, $\varepsilon_{\text {ref }}$ and a perturbed part confined inside the thin film medium. Hence the dielectric function of the system can be expressed as

$$
\varepsilon_{p}(z)=\varepsilon_{r e f}+\Delta \varepsilon_{p}(z)
$$

The assumption strictly depends on the nature of the medium being investigated. With equation (2), equation (1) can be written as

$$
\nabla^{2} \psi(z)+\left[k_{o}^{2} \varepsilon_{r e f}-k_{o}^{2} \Delta \varepsilon_{p}(z)\right]=0
$$

Where we define the term, $V(z)$ containing the dielectric perturbation, $\Delta \varepsilon_{p}(z)$ as

$$
V(z)=-k_{o}^{2} \Delta \varepsilon_{p}(z)
$$

with $k_{o}^{2}=\mu_{0} \varepsilon_{0} \omega_{0}$

We now write equation (3) thus

$$
\nabla^{2} \psi(z)+k_{0}^{2} \varepsilon_{r e f} \psi(z)=0
$$

if we consider the $-k_{o}^{2} \Delta \varepsilon_{p}(z)$ as a source function $f^{\prime}(z)$

The Green's function associated to equation (5) is

$$
\left[\nabla^{2}+\gamma\right] G\left(z, z^{\prime}\right)=\delta\left(z, z^{\prime}\right)
$$

The solution of equation (6) is given as

$$
G\left(z, z^{\prime}\right)=\left\{\begin{array}{l}
A\left(z^{\prime}\right) \sin \gamma z+B\left(z^{\prime}\right) \cos \gamma z ; a \leq z \leq z^{\prime} \\
C\left(z^{\prime}\right) \sin \gamma z+D\left(z^{\prime}\right) \cos \gamma z ; z^{\prime} \leq z \leq a
\end{array}\right.
$$


To eliminate the arbitrary constants, $G\left(z, z^{\prime}\right)$ must satisfy a given boundary condition that $G(z, 0)$ $=G(z, a)$ Coupled with the fact that at $z=z^{\prime}$, the derivative of $G\left(z, z^{\prime}\right)$ must be continuous.

This will now lead the solution to be

$$
G\left(z, z^{\prime}\right)=\left\{\begin{array}{l}
\frac{\sin \gamma z^{\prime} \sin (a-z)}{\gamma \sin \gamma a} ; a \leq z^{\prime} \leq z \\
\frac{\sin \gamma z \sin \left(a-z^{\prime}\right)}{\gamma \sin \gamma a} ; z \leq z^{\prime} \leq a
\end{array}\right.
$$

Having obtained the Green's function associated with the problem, the solution of the inhomogeneous equation becomes

$$
\psi(z)=\int_{0}^{a} G(z) f^{\prime}\left(z^{\prime}\right) d z^{\prime}
$$

Based on this, we now spell out the total field propagation through the thin film

$$
\psi(z)=\psi^{o}(z)+\int_{0}^{a} G\left(z, z^{\prime}\right) V\left(z^{\prime}\right) \psi(z) d\left(z^{\prime}\right)[\text { Martin et al, 1994] }
$$

This is known as the integral form of Lippmann-Schwinger equation whose counterpart, Dyson's equation is given as

$$
G\left(z, z^{\prime}\right)=G^{o}\left(z, z^{\prime}\right)+\int_{0}^{a} G\left(z, z^{\prime}\right) V\left(z^{\prime}\right) G\left(z, z^{\prime}\right) d z^{\prime}[\text { GAO, 2005; Ugwu, 2011](11) }
$$

This equation is meant for use in iterating Green's function, $G\left(z, z^{\prime}\right)$ over the thin film medium for subsequent use in equation (10) in order to obtain the value of the propagating field, $\psi(z)$ over the same thin film medium. Because of the implicit nature of these equations (10) and (11), a grid is defined over the system in which discretization procedure preferably variable Gaussian grid, $\Delta_{k}$ is applied on the deposited thin film. We suppose that the discretized system contains $\mathrm{N}$ meshes from which $\mathrm{Np}$ describes the perturbation $(\mathrm{Np} \leq \mathrm{N})$. We designate the discretized field the Green operator and the perturbation by $\psi_{i}, G_{i, j}$ and $V_{i}$, respectively. The discretized form of equations (10) and (11) are

$$
\begin{aligned}
& \psi_{i}=\psi_{i}^{o} \sum_{k=1}^{N_{p}} G_{i, k}^{0} V_{k} \Delta_{k} \psi_{k} \\
& G_{i, j}=G_{i, j}^{0}+\sum_{k=1}^{N_{p}} G_{i, k}^{o} V_{k} \Delta_{k} G_{k, j}
\end{aligned}
$$

However the direct numerical resolution of equation (12) is particularly time consuming and lead to numerical instabilities because of the singular behaviour of $G_{i, k}^{o}$ result of this, an iterative scheme for computing the field propagating through the film is carried out by introduction of the Dyson's equation as already stated in equation (11) and (13) as it yields the Green's function, $G^{o}\left(z, z^{\prime}\right)$ of the homogeneous reference system.[Martin et al, 1994]. By applying this scheme, the number of operations and the size of the matrix $G_{i, j}$ and $\psi_{i}$ quired for every step can be clearly spelt out, For instance for three meshes we have 


$$
\begin{aligned}
& \mathrm{G}_{1,1}=\mathrm{G}_{1,1}^{\mathrm{o}}+\mathrm{G}_{1,1}^{\mathrm{o}} \mathrm{V}_{1} \Delta_{1} \mathrm{G}_{1,1}+\mathrm{G}_{2,2}^{\mathrm{o}} \mathrm{V}_{2} \mathrm{G}_{2,1}+\mathrm{G}_{1,3}^{\mathrm{o}} \mathrm{V}_{3} \Delta_{3,1} \\
& \mathrm{G}_{2,1}=\mathrm{G}_{2,1}^{\mathrm{o}}+\mathrm{G}_{2,1}^{\mathrm{o}} \mathrm{V}_{1} \Delta_{1} \mathrm{G}_{1,1}+\mathrm{G}_{2,2}^{\mathrm{o}} \mathrm{V}_{2} \Delta_{2} \mathrm{G}_{2, \mathrm{I}}+\mathrm{G}_{2,3}^{\mathrm{o}} \mathrm{V}_{3,} \Delta_{2} \mathrm{G}_{3,1} \\
& \mathrm{G}_{3,1}=\mathrm{G}_{3,1+}^{\mathrm{o}} \mathrm{G}_{3,1}^{\mathrm{o}} \mathrm{V}_{1} \Delta_{1} \mathrm{G}_{1,1}+\mathrm{G}_{3,2}^{\mathrm{o}} \mathrm{V}_{2} \Delta_{2,1}+\mathrm{G}_{3,3}^{\mathrm{o}} \mathrm{V}_{3} \Delta_{3} \mathrm{G}_{3,1} \\
& \mathrm{G}_{1,2}=\mathrm{G}_{1,2}^{\mathrm{o}}+\mathrm{G}_{1,1}^{\mathrm{o}} \mathrm{V}_{1} \Delta_{1} \mathrm{G}_{1,2}+\mathrm{G}_{1,2}^{\mathrm{o}} \mathrm{V}_{2} \Delta_{2} \mathrm{G}_{2,2}+\mathrm{G}_{1,2}^{\mathrm{o}} \mathrm{V}_{3} \Delta_{3} \mathrm{G}_{3,2} \\
& \mathrm{G}_{2,2}=\mathrm{G}_{2,2}^{\mathrm{o}}+\mathrm{G}_{2,1}^{\mathrm{o}} \mathrm{V}_{1} \Delta_{1,2} \mathrm{G}_{1,2}+\mathrm{G}_{2,2}^{\mathrm{o}} \mathrm{V}_{2} \Delta_{2} \mathrm{G}_{2,2}+\mathrm{G}_{2,3} \mathrm{~V}_{3} \Delta_{3} \mathrm{G}_{3,2} \\
& \mathrm{G}_{3,2}=\mathrm{G}_{3,2}^{\mathrm{o}}+\mathrm{G}_{3,1} \mathrm{~V}_{1} \Delta_{1} \mathrm{G}_{1,2}+\mathrm{G}_{3,2}^{\mathrm{o}} \mathrm{V}_{2} \Delta_{2} \mathrm{G}_{2,2}+\mathrm{G}_{3,3}^{\mathrm{o}} \mathrm{V}_{3} \Delta_{3} \mathrm{G}_{3,2} \\
& \mathrm{G}_{1,3}+\mathrm{G}_{1,3}^{\mathrm{o}}+\mathrm{G}_{1,1}^{\mathrm{o}} \mathrm{V}_{1} \Delta_{1} \mathrm{G}_{1,3}+\mathrm{G}_{1,2}^{\mathrm{o}} \mathrm{V}_{2} \Delta_{2} \mathrm{G}_{2,3}+\mathrm{G}_{1,3} \mathrm{~V}_{3} \Delta_{3} \mathrm{G}_{3,3} \\
& \mathrm{G}_{2,3}+\mathrm{G}_{2,3}^{\mathrm{o}}+\mathrm{G}_{2,1} \mathrm{~V}_{1} \Delta_{1} \mathrm{G}_{1,3}+\mathrm{G}_{1,2}^{\mathrm{o}} \mathrm{V}_{2} \Delta_{2} \mathrm{G}_{2,3}+\mathrm{G}_{2,3}^{\mathrm{o}} \mathrm{V}_{3} \Delta_{3} \mathrm{G}_{3,3} \\
& \mathrm{G}_{3,3}=\mathrm{G}_{3,3}^{\mathrm{o}}+\mathrm{G}_{3,1}^{\mathrm{o}}+{ }_{1} \mathrm{~V}_{1} \Delta_{1} \mathrm{G}_{1,2}+\mathrm{G}_{3,2}^{\mathrm{o}} \mathrm{V}_{3} \Delta_{3} \mathrm{G}_{2,3}+\mathrm{G}_{3,3}^{0} \mathrm{~V}_{3} \Delta_{3} \mathrm{G}_{3,3}
\end{aligned}
$$

Equation (i) to (ix) represent equation (14) that are the nine algebraic equations, that will lead to nine values of $\mathrm{G}_{\mathrm{i}, \mathrm{j}}$ as given below

$$
\mathrm{G}_{1,1}, \mathrm{G}_{1,2}, \mathrm{G}_{1,3}, \mathrm{G}_{2,1} \mathrm{G}_{2,2} \mathrm{G}_{3,2} \mathrm{G}_{1,3} \mathrm{G}_{23} \mathrm{G}_{3,3} \text {. }
$$

Equation (14) is written in a compact matrix form as shown in equation (16) and from that the algebraic equation corresponding to $\psi_{i}$ are obtained thus

$$
\begin{aligned}
& \Psi_{1}=\Psi_{\mathrm{i}}+\mathrm{G}^{\mathrm{o}}{ }_{1,1}, \mathrm{~V}_{1} \Delta_{1} \Psi_{1}+\mathrm{G}_{1,2}^{\mathrm{o}} \mathrm{V}_{2} \Delta_{2} \Psi_{2}+\mathrm{G}^{\mathrm{o}}{ }_{1,3} \mathrm{~V}_{3} \Delta_{3} \Psi_{3} \\
& \Psi_{2}=\Psi_{3}^{\mathrm{o}}+\mathrm{G}_{3,1}^{\mathrm{o}} \mathrm{V}_{1} \Delta_{1} \Psi_{1}+\mathrm{G}_{3,2}^{\mathrm{o}} \mathrm{V}_{2} \Delta_{2} \Psi_{2}+\mathrm{G}_{3,3}^{\mathrm{o}} \mathrm{V}_{3} \Delta_{3} \Psi_{3} \\
& \Psi_{3}=\Psi_{3}^{\mathrm{o}}+\mathrm{G}_{3,1}^{\mathrm{o}} \mathrm{V}_{1} \Delta_{1} \Psi_{1}+\mathrm{G}_{3,2}^{\mathrm{o}} \mathrm{V}_{2} \Delta_{2} \Psi_{2}+\mathrm{G}_{3,3}^{\mathrm{o}} \mathrm{V}_{3} \Delta_{3} \Psi_{3}
\end{aligned}
$$

These equations could be written in a compact matrix form as in the equation below.

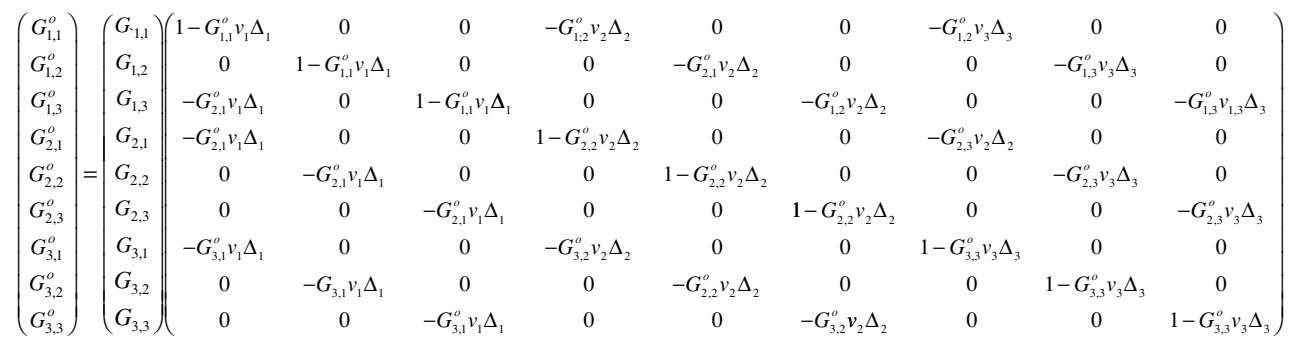

of which the corresponding field matrix counterpart from algebraic equation (15) is given below.

$$
\left(\begin{array}{ccc}
1-G^{o}{ }_{1,1} V_{1} \Delta_{1} & -G^{o}{ }_{1,2} V_{2} \Delta_{2} & -\boldsymbol{G}_{1,3}^{o} V_{3} \Delta_{3} \\
-G_{2,1}^{o} V_{1} \Delta_{1} & 1-G^{o}{ }_{2,2} V_{2} \Delta_{2} & \Delta-G^{o}{ }_{2,3} V_{3} \psi_{3} \\
-G^{o}{ }_{3,1} V_{1} \Delta_{1} & -G^{o}{ }_{3,2} V_{2} \Delta_{2} & \boldsymbol{I}-\boldsymbol{G}_{3,3}^{o} \nu_{3}-G_{3,2}^{o} V_{2} \Delta_{3}
\end{array}\right)\left[\begin{array}{l}
\psi_{1} \\
\psi_{2} \\
\psi_{3}
\end{array}\right]=\left[\begin{array}{c}
\psi_{1}^{0} \\
\psi_{2}^{0} \\
\psi_{3}^{0}
\end{array}\right]
$$


Based on this we used five and eight meshes in our solution respectively and we were able to obtain five and eight values of field respectively that enable us to obtain the result as shown in the Figures 1 to 6

\section{RESULT AND DISCUSSION}

Discretized form of Lippmann-Schwinger equation and its counterpart, Dyson's equation as shown in equation [12] and [13] were introduced based on the fact that the numerical approximation involved in handling [12] and [13] were easier due to the difficulty imposed by direct integration of equations [10] and [11] because of their implicit nature. The mesh size, $\Delta_{k}$ and the discretized format, $\mathrm{N}$ meshes introduced in formulation enhanced the computation. For instance, the number of processes involved in the computation of the field, $\Psi_{\mathrm{i}}$ depends on the number of the meshes considered. When $N_{p}$ meshes are considered out of $\mathrm{N}, \mathrm{N}$ algebraic equations interms of Green's function, $G_{i, j}$ are obtained which in turn lead $\mathrm{N} \times \mathrm{N}$ matrix. The computed Green's function are used in equation [12] to obtain the value of the field, $\Psi_{\mathrm{i}}$. as shown in Fig. 1 to Fig. 6. The result show that Fig. 1 and Fig. 2 and Fig. 3 depict variation of Green, s Function, $G_{i, j}$ against the field, $\Psi_{\mathrm{i}}$ has positive correlation 0f 0.993 , and 0.944 for visible and ultraviolet region of electromagnetic wave for $N_{p}=5$ and 8 respectively. While for infrared as shown in Fig. 3, the correlation is negative, -0.985 for $N_{p}=8$. The field variation with both thickness and wavelength for $N_{p}=5$ and 8 were seen to be $0.596,0.993$ as shown

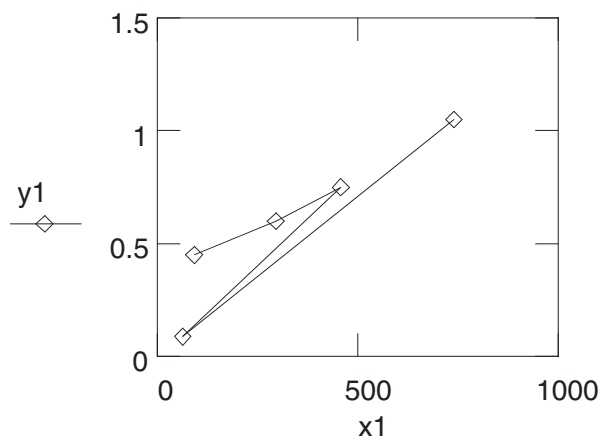

Figure 1: Variation of Green's function $\mathrm{G}_{\mathrm{i}, \mathrm{j}}(\mathrm{y} 1)$ gainst Field value, $\psi_{\mathrm{i}}(\mathrm{x} 1)$ for ultraviolet region of Electromagnetic wave when $N_{p}=5$

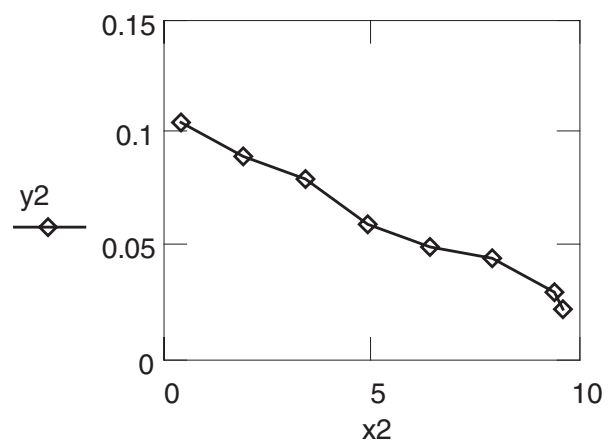

Figure 2: Variation of Green's function, $G_{i, j}(\mathrm{y} 2)$ against Field value $\psi_{\mathrm{i}}(\mathrm{x} 2)$ for visible region of Electromagnetic wave when $N_{p}=8$ 


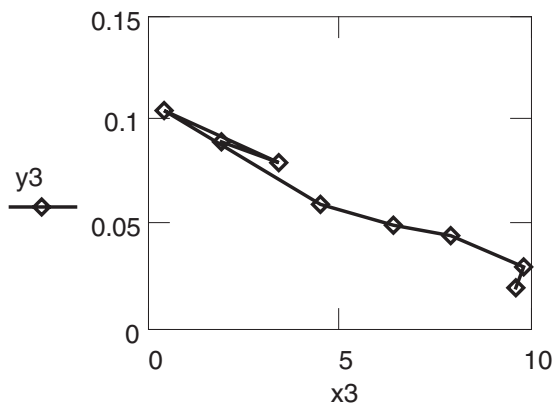

Figure 3: Variation of Green's function $G_{i, j}(\mathrm{y} 3)$ against Field value $=\psi_{\mathrm{i},}(\times 3)$ for infrared region of Electromagnetic wave when $N_{p}=8$

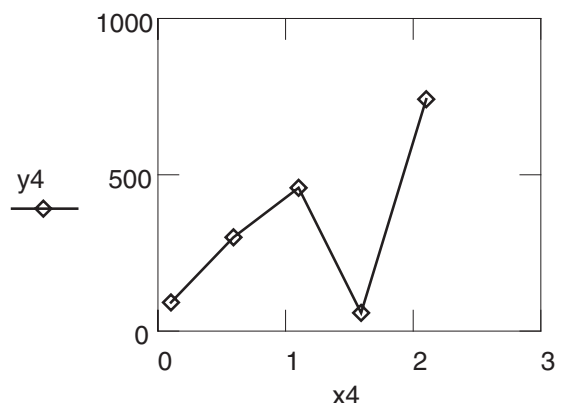

Figure 4: Variation of Green function $\mathrm{G}_{\mathrm{i}, \mathrm{j}}(\mathrm{y} 4)$ against Field value, $\psi_{\mathrm{i},}(\mathrm{x} 4)$ for infrared region of Electromagnetic wave when $N_{p}=5$

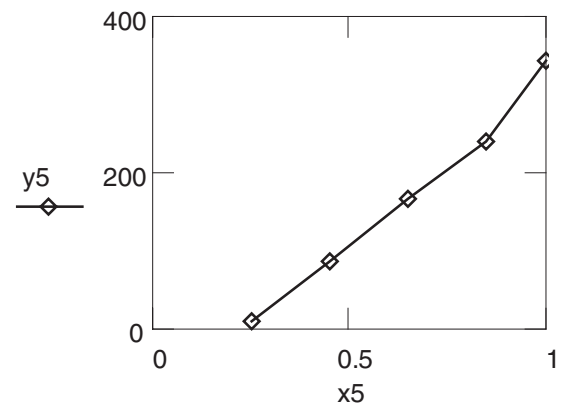

Figure 5: Variation Field value $\psi_{\mathrm{i}}(\mathrm{y} 5)$ against wavelength $\lambda \mu \mathrm{m}(\mathrm{x} 5)$ When $N_{p}=5$

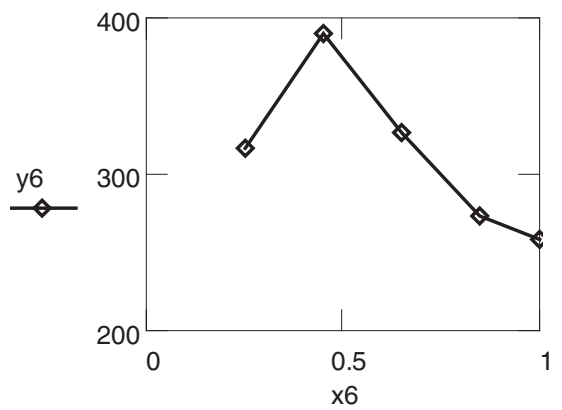

Figure 6: Variation of perturbation $\mathrm{V}(\mathrm{z})(\mathrm{y} 6)$ against wavelength $\lambda \mu \mathrm{m}(\mathrm{x} 6)$, when $N_{p}=5$ 
in Fig. 4 and 5 respectively. While that of Fig. 6 that relates Perturbation, $V(z)$ and Wavelength for $N_{p}=5$ depicts negative correlation

\section{CONCLUSION}

We have presented an approach to the computation of field propagation in dielectric thin film medium known as iterative scheme involving the use of Green's Function technique. Lippmann-Schwinger equation and its counterpart Dyson' equation was used to obtain the solution of the wave equation. In this approach, unlike the case of direct integration, no approximation as a result of the implicit nature of the equation was invoked because of the introduction of the discretization terms. The influence of the numerical parameters such as dielectric perturbation and number of meshes on the Green's function correlation on Field value was examined. Finally, the iterative scheme based on the parallel use of LippmannSchwinger and Dyson' equation were judiciously used and found to be useful in the computation

\section{REFERENCE}

[1] Feit, M.D and Fleck Jr. J.A (1978) Light propagation in graded- index optical fibres Appl. Optic 17 3990-3998

[2] Feit, M.D and Fleck Jr. J.A (1979) Calculation of dispersion in graded-index multimode fibres by propagating beam method. Appl. Optics 18 2843-2851

[3] Feit, M.D and Fleck Jr. J.A (1980) Computation of mode properties in optical fibre waveguides by a propagating beam method. Appl. Optics 19 1154-1164

[4] van Roey, J. van der Donk J. and Lagasse P.E (1981) beam Propagation method; analysis and assessment. J. Opt Soc. Am. 71 803- 810

[5] Yevick, D and Glasner, M (1990), Forward wide- angle light propagation in semiconductor rib waveguides. Opt. lett. 15 174-176

[6] Splett A, M. Majd and K. Petermann (1991) A novel beam propagation method for large refractive index steps and large propagation distances. IEEE. Photon, Technol. Lett. 3 466-468

[7] Stern, M.S (1991) Rayleigh quotient solution of semivectorial field problem for optical waveguides with arbitrary index profiles IEEE Proc. J. 138 185-190

[8] Clauberg, R and P. van Allmen(1991) Vectorial beam-propagation method for integrated optics. Elecron Lett. 27 654-655

[9] Huang, W.P: Xu, C.L, Chu, S.T and S.K Chaudhuri (1991) A vector beam propagation method for guided wave optics. IEEE Photon Technol. Lett. 3 910- 913

[10] Martin, O.J.F, Clauberg, R. and P. van Allmen Demonstration of the three-dimensional vectorial beam propagation; presented at the European conference on optical communication, Paris Sept. 9-12, 1991

[11] Chung.Y.Dagli, N.and and Thyle/n(1991) Explicit finite- diference vectorial beam propagation method Electron Lett. 27 2119-2120

[12] Chuang, W; Xu, C. Chu, S.T and Chaudhuri, S.K [1992] The finite-difference vector beam propagation method. Analysis and assessment. IEEE. J.Ligth wave Technol. 10 295-305

[13] Liu, J. M and Gomelsky, L. (1990)Vectorial beam propagation method. J Opt. Soc Am A9 1574-1585

[14] Martin J.F.O, Alian Dereux and Girard Chirstian (1994) Iterative scheme for computing exactly the total field propagating in dielectric structure of arbitrary shape.J. Opt. Soc. Am vol. 113 1073-1081 
[15] Ugwu E.I Uduh P.C and Agbo G. A ( 2007) The effect of change in refractive index on wave propagating through $\left(\mathrm{FeS}_{2}\right)$ thin film. J. Appl Sci 7(4) 570-574

[16] Ugwu, E.I ( 2011)computational method of studying the field propagation through an inhomogeneous thin film using Lippmann-Schwinger equation. Trend in Appl Science Research 6(1) $73-80$

[17] Gao, G. Torres-Verdin, C. and Habashy, T.M (2005) Analytical techniques to evaluate the integrals of 3D and2D spatial, Dyadic Green's functions. Progress in Electromagnetic Research, PIER 52 47-80 